\title{
Lapurdum
}

Euskal ikerketen aldizkaria | Revue d'études basques |

Revista de estudios vascos | Basque studies review

$12 \mid 2008$

Numéro XII

\section{U-Z [texte intégral]}

U.D.A. Ustaritz d'abord, U.R.S.S.A.F. de \{la région de\} Bayonne, U.R.S.S.A.F.-info, Ufakoa, Uhaitza, Union (L') de la gauche " informe », Union départementale des syndicats de l'industrie hôtelière des BassesPyrénées

\section{OpenEdition \\ Journals}

Édition électronique

URL : http://journals.openedition.org/lapurdum/1655

DOI : 10.4000/lapurdum. 1655

ISSN : 1965-0655

Éditeur

IKER

Édition imprimée

Date de publication : 1 février 2008

Pagination : 161-170

ISBN : 978-2-86781-617-8

ISSN : 1273-3830

Référence électronique

«U-Z [texte intégral] », Lapurdum [En ligne], 12 | 2008, mis en ligne le 24 mai 2010, consulté le 01 mai 2019. URL : http://journals.openedition.org/lapurdum/1655; DOI : 10.4000/lapurdum. 1655

Ce document a été généré automatiquement le 1 mai 2019.

Poitelon J.C. | IKER 


\section{U-Z [texte intégral]}

U.D.A. Ustaritz d'abord, U.R.S.S.A.F. de \{la région de\} Bayonne, U.R.S.S.A.F.-info, Ufakoa, Uhaitza, Union (L') de la gauche « informe », Union départementale des syndicats de l'industrie hôtelière des BassesPyrénées

\section{U.D.A. Ustaritz d'abord voir Ustaritz d'abord}

2 U.R.S.S.A.F. de \{la région de\} Bayonne. Eléments statistiques. Annuel. 1995 - $1999\left(\mathrm{n}^{\circ}\right.$ 1-5). 2002-2003. - Anglet, 30 x 21 puis 21 x $15 \mathrm{~cm}$

A partir de 2002 porte en tête: U.R.S.S.A.F. Aquitaine. - En français. - Publ. par: U.R.S.S.A.F. [Union de recouvrement des cotisations de sécurité sociale et d'allocations familiales] de la région de Bayonne [Anglet]

3 U.R.S.S.A.F.-info. Bulletin de liaison avec les entreprises. juin 1999 ( $\left.{ }^{\circ} 1\right)$ [?]. - Anglet, $30 \times 21 \mathrm{~cm}$

En français. - Publ. par: U.R.S.S.A.F. [Union de recouvrement des cotisations de sécurité sociale et d'allocations familiales] de la région de Bayonne [Anglet]

Ufakoa [Bayonne] voir Revue (La) Ufakoa

5 Ufakoa. Euskal dantzarien biltzarra, danses et musiques traditionnelles [puis danses et musiques du Pays basque]. Trimestriel puis irrégulier. févr./avr. $1998\left(\mathrm{n}^{\circ} 1\right) \rightarrow$. Hiriburu puis Luhuso, $21 \times 15$ puis $30 \times 21 \mathrm{~cm}$

En français; quelques textes basques. - Publ. par: Euskal dantzarien biltzarra [Saint-Pierred'Irube puis Louhossoa]. - Remplace: Euskal dantzarien biltzarra... Activités

Uhaitza. Bulletin d'information. avr. 1994 - juil. 1995 (n¹-3). - Menditte, 30 x $21 \mathrm{~cm}$ En français; quelques textes basques. - Remplacé par: Lettre (La) des associations en Soule

7 Union (L') de la gauche « informe ». Lettre. Irrégulier. [janv. 1978 - janv. 1980] [?] ( ${ }^{\circ}$ 1-11). mai $1981\left(\mathrm{n}^{\circ} 12\right)$. - [Mouguerre], 30 x $21 \mathrm{~cm}$

En mai 1981 a paru sous le titre: Lettre d'union de la gauche. - En français. - Publ. par les membres de la "Liste d'union de la gauche» après les élections municipales de mars 1977 et jusqu'aux élections partielles de déc. 1979. - Ssuite de: Lettre aux électeurs

Union départementale des syndicats de l'industrie hôtelière des Basses-Pyrénées. Bulletin d'information. Mensuel. 28 févr.-juin 1946 ( $\left.{ }^{\circ} 1-5\right)$. - Biarritz, 28 x $22 \mathrm{~cm}$ 
En français. - Devient: Fédération «Pyrénées-Sud-Ouest» des syndicats de l'industrie hôtelière... Bulletin d'information

Union (L') des peuples de l'Hexagone. \{Bulletin bimestriel\}. mars 1983 - oct./déc. 1984 $\left(n^{\circ} 1-9\right)$. - Bayonne, 30 x $21 \mathrm{~cm}$

En français. - Publ. par: Union des peuples de l'Hexagone (U.P.H.) [Bayonne]

Union locale C.G.T. de Bayonne. En direct avec vous voir En direct avec vous

$11{ }^{*}$ Union (L') locale P.E.E.P. Bulletin de liaison et d'informations des associations de parents d'élèves des établissements primaires et secondaires du Pays basque et du BasAdour. sept.1972 - avril 1975 ( ${ }^{\circ} 1$ - 9) [?]. - Bayonne

En français. - Reprend les éléments de: Echo (L') des parents

Union nationale des combattants. Groupe basque. Anciennement « Le Poilu basque ». Journal [puis Bulletin] trimestriel de l'U.N.C. - U.N.C.A.F.N. [puis Union nationale des combattants... Bulletin trimestriel d'information de « l'Union nationale des combattants, Groupe basque »]. $3^{\mathrm{e}}$ trim. $1986\left(43^{\mathrm{e}}\right.$ année, $\left.\mathrm{n}^{\circ} 385\right) \rightarrow$. Bayonne, $43 / 45 \times 32 \mathrm{~cm}$

En français. - Suite de: Poilu (Le) basque

Union nationale des combattants. La Lettre du Trait d'union voir Lettre (La) du Trait d'union

Union nationale des combattants. Trait d'union voir Trait d'union. Bulletin d'information, Section d'Anglet... et Trait d'union. Supplément...

Union pour la démocratie française. La Lettre de l'U.D.F. voir Lettre (La) de l'U.D.F.

Units toustem voir Atchiki

Urcuit. Bulletin municipal. Irrégulier puis semestriel ou annuel. [mai 1983] (n¹) - [...]. juil./ déc. 1989 - janv./juil.1994 (n¹-10). janv. 1996 - déc. 2000 (n¹-6). - Urcuit, 21 x 15 puis $30 \times 21 \mathrm{~cm}$

Avec un n'spécial d'oct. 1997. - En janv./juil. 1990 a paru sous le titre: Quoi de neuf ?; de juil.1990/janv.1991 à janv./juil. 1992 a paru sous le titre: Journal (Le) de la commune. - En français. - Devient: Urcuit. Revue municipale

Urcuit. La vie municipale. [oct. 1997- janv. 1998] (2 nº). juin 1999. - [Urcuit], 30 x $21 \mathrm{~cm}$

En juin 1999 a paru sous le titre: Urcuit. Lettre de l'opposition. - En français

Urcuit. Lettre de l'opposition voir Urcuit. La vie municipale

Urcuit. Revue municipale. Annuel. 2002 [?] $\rightarrow$. Urcuit, 30 x $21 \mathrm{~cm}$

En français. - Suite de: Urcuit. Bulletin municipal. - Paraît parallèlement à: Grain (Le) de sel. Bulletin de liaison d'Urcuit

Urketa 2000. Information et solidarité. Jakin eta elkartasuna. Trimestriel. hiver-été 1987 (n¹-3). - Urcuit, $30 \times 21 \mathrm{~cm}$

En basque et français

Urrugne. Bulletin des élus de gauche voir Bulletin de liaison de la liste d'union de la gauche...

Urrugne. Bulletin municipal. Irrégulier. mai 1978.1984.1989 [?]. - Urrugne, 30 x $21 \mathrm{~cm}$

En mai 1978 a paru sous le titre: Mairie d'Urrugne. Bulletin d'information. - En français. Devient: Urruñan bizi

Urrugne. Informations municipales. Irrégulier. juil. 1994 - mai 1996 (n¹-13). oct. 1997 nov. 2000 ( $\left.{ }^{\circ} 14-26\right)$ [?]. - Urrugne, 30 x $21 \mathrm{~cm}$ 
Jusqu'en févr. 1996 a paru sous le titre: Informations municipales. - En français. - Parait parallèlement à: Urruñan bizi

Urrugne. Journal d'information de la gauche [puis d'information des élus de gauche]. sept. 1995 - févr. $1997\left(3 \mathrm{n}^{\circ}\right)$. févr. 2000 [?]. - Urrugne, 30 x $21 \mathrm{~cm}$

En français. - Suite de: Bulletin de liaison de la liste d'union de la gauche, Urrugne

Urrugne demain voir Bihar Urruñan

* Urrugne-infos. Bulletin d'information des communistes d'Urrugne. $3 n^{\circ}$ par an. janv. 1998 - janv. 2002 (n॰11]-13). - [Urrugne], 30 x $21 \mathrm{~cm}$ En français. - Publ. par: Parti communiste français. Cellule d'Urrugne [Urrugne]

Urruñan bizi. Bulletin d'informations [puis Bulletin municipal]. Irrégulier. mai 1992 ( $\left.{ }^{\circ} 1\right)$ - 1994 [?]. mai $1996\left(\mathrm{n}^{\circ} 1\right) \rightarrow$. Urrugne, 30 x 21 ou 42 x $30 \mathrm{~cm}$

Avec un nªnnuel "spécial budget» de 1997 à 2001 [?]. - Titre français: Vivre à Urrugne; en mai 2001 a paru sous le titre: Vivre Urruñan bizi. - En français. - Suite de: Urrugne. Bulletin municipal. - Jusqu'en 2000 paraît parallèlement à: Urrugne. Informations municipales

Urt. Bulletin municipal. Informations \{et\} communication. Trimestriel. oct.1995 (n51) $\rightarrow$. Urt, $30 \times 21 \mathrm{~cm}$

En français. - Suite de: Bulletin municipal d'information. Mairie d'Urt

* Urt, Urcuit, Lahonce. Bulletin d'informations municpales. déc. 1987 ( $\left.n^{\circ} 1\right)$. - [Urcuit], $21 \times 15 \mathrm{~cm}$

Bulletin intercommunal. - En français

Ustaritz autrement. déc.1995 - juin 1996 (n¹-2). - [Ustaritz], 30 x $21 \mathrm{~cm}$

Avec un $n^{\circ}$ spécial de mars 1997. - En français

Ustaritz d'abord. Feuillet \{de\} liaison. janv. 2002 - mars 2003 (n¹-2). - Ustaritz, $30 \times 21 \mathrm{~cm}$

Porte en tête: U.D.A. - En français. - Publ. par: Ustaritz d'abord [Ustaritz]

Ustaritz-Jatxou. Rugby-club voir Rugby-club Ustaritz-Jatxou. Saison

Ustaritz-Uztaritze \{,capitale du Labourd\}. Bulletin municipal d'information. Irrégulier. oct. 1990 - févr. 1994 (n¹-5). janv. $1995 \rightarrow$. Ustaritz, 30 x $21 \mathrm{~cm}$

Le sous-titre varie et disparaît. - En français

Utopia-cinéma [ou Utopia-Bayonne-cinéma]. Gazette. Mensuel ou irrégulier. 6 juil./2 août 1983 - 12 avr./9 mai 1989 (n¹-69) [?]. - Bayonne, 30 x $21 \mathrm{~cm}$

En français. - Remplacé par: Atalante (L')-cinéma

Uztaritz-écho. oct. 1993 - [janv. 1994] (n¹-2) [?]. - [Ustaritz], 30 x 21 cm

En basque et français. - Publ. par: Zergatikote [Ustaritz]

Uztaritze village vivant voir Herria bizi dadin

V.A.M. Vélo, auto, moto, Landes-Pyrénées. Mensuel. [...]. - Ciboure, 30 x $21 \mathrm{~cm}$

Paraissait en sept. 1985 ( $\left.n^{\circ} 6\right)$. - En français

Vacances et famille. Bulletin de liaison. Bulletin périodique d'information de l'association Vacances et famille. 1957 - [...] . - Guéthary, 42 x $31 \mathrm{~cm}$

Paraissait en janv. 1958 (2e année, n³). - En français

Vent (Du)... \{Mensuel [puis Bimestriel] pensé, conçu et réalisé par la M.J.C. de Balichon, Bayonne\}. Mensuel puis bimestriel puis irrégulier. avr.-mai/juin 1968 (n¹-2). févr.1981 [juin] 1994 (n.s. n¹-78). - Bayonne, 27 x 21 puis 30 x $21 \mathrm{~cm}$ 
En français. - Publ. par: Maison des jeunes et de la culture de Balichon [Bayonne]. Remplacé par: Lettre (La) du citoyen

Vents et marées. Trimestriel. mars 1977 - juin 1995 (n¹-60). - Anglet, 30 x $21 \mathrm{~cm}$

Avec un n $n^{\circ} 0$ de déc. 1976 et de nombreux n hors série, dont certains ont donné lieu à plusieurs rééd. - En français. - Publ. par: Association revue poétique «Vents et marées» [Anglet]. - En mai 1977 un groupe de collaborateurs quitte la revue et fonde: Muscade. - Le n'40 n'a pas paru Vérité (La) voir Egia. Bulletin d'information de l'opposition municipale

Vie (La) de Biarritz. Bulletin municipal [puis Bulletin municipal \{et\} d'informations locales puis Bulletin d'informations locales]. Mensuel. juil.1977 - juin 1991 ( $\left.{ }^{\circ} 1-163\right)$. juil.1991-déc. 1991/janv. 1992 (n4-7). - Biarritz, 29 x 21 puis 31 x $22 \mathrm{~cm}$ Avec des nospéciaux de févr.1983 et févr.1989 intitulés: 1977 - 1983. Bilan d'un mandat et 1983 1989. Un bilan pour Biarritz. - En français. - Remplace: Biarritz. Revue municipale. - Après le départ de Bernard Marie de la mairie de Biarritz en mars 1991, remplacé comme bulletin municipal par: Biarritz magazine; à partir d'avril 1991 continue à paraître comme bulletin politique, sous le titre (à partir de mai): Vie (La) de Biarritz et du Pays basque. - Fusionne avec: Echos (Les) de Biarritz pour former: Vie (La) et les Echos de Biarritz et du Pays basque

Vie (La) de Donazaharre. Bulletin municipal. Annuel. déc. $1995\left(n^{\circ} 1\right) \rightarrow$. Saint-Jean-leVieux, $30 \times 21 \mathrm{~cm}$ En français

Vie (La) de Jatxou voir Jatsun zer berri

Vie (La) des Biarrots. La Vie des Biarrots est éditée par les conseillers municipaux du groupe «Biarritz avec vous» [puis par l'association «Biarritz avec vous»]. Irrégulier. juin $2001\left(\mathrm{n}^{\circ} 1\right) \rightarrow$. Biarritz, 30 x $21 \mathrm{~cm}$

En français. - Reprend les éléments de: Biarritz avec vous

47 Vie (La) et les Echos de Biarritz et du Pays basque. Mensuel. mars 1992 - janv./févr. 1995 (n¹-29). - Biarritz, 30 x $21 \mathrm{~cm}$

Partie du titre en gros caractères: Pays basque. - En français. - Dir. publ. Michèle Alliot-Marie ou Bernard Marie. - Formé par la fusion de: Vie (La) de Biarritz et Echos (Les) de Biarritz

municipale. Annuel. [nov. 1989] $\rightarrow$. La Bastide-Clairence, 30 x $21 \mathrm{~cm}$

En [nov. 1989] a paru sous le titre: Bulletin municipal d'informations. - A partir d'août 1992 porte en tête: La Bastide-Clairence. - En français. - Suite de: Bulletin municipal de La BastideClairence

* Vie ovale. \{Le magazine du Biarritz olympique Pays basque\}. Au cœur et autour du rugby à Biarritz et au Pays basque. Bimestriel. nov. 2004 (saison 2004/05, nº $\rightarrow \rightarrow$. Paris, Biarritz, 30 × $23 \mathrm{~cm}$

Avec un $n^{\circ}$ hors série de [sept. 2004]. - En français. - Voir aussi Biarritz olympique... et Rouge et blanc

50 Vie (La) propre voir Bizi garbia

Viens... suis-moi [puis Viens, suis-moi]. \{Bulletin de l'œeuvre des vocations \{du\} Diocèse de Bayonne\}. Trimestriel ou irrégulier. avr. 1946. déc. $1946\left(23^{e}\right.$ année, $\left.n^{\circ} 1\right) \rightarrow$. Bayonne puis Pau puis Bayonne, de 22 x 14 à 26 x $19 \mathrm{~cm}$

En français; à partir de 1972 quelques textes basques. - Suite de: Bulletin trimestriel de l'œuvre des vocations du Diocèse de Bayonne [oct.1924 - mai 1940] 

nov. 2000 ( $\left.n^{\circ} 1-10\right)$. - Saint-Palais, 30 x $21 \mathrm{~cm}$ Titre basque: Euskal herrian: Baxe-Nafarroko eta Xiberoko berriak. - En français Landes et Pyrénées-Atlantiques. [janv.2001] - avr.2002 (n¹-5). - Anglet, 30 x $21 \mathrm{~cm}$ En français. - Publ. par: Association des pensionnés de la marine marchande et de la pêche des Landes et Pyrénées-Atlantiques [Anglet] Villa-expo-infos. nov./déc.2002 - printemps/été 2003 (n¹-2). - Biarritz, 30 x $21 \mathrm{~cm}$ En français. - Publ. par la Ville de Biarritz Villa Pia (Le) enchaîné. [automne 1985] - juin 1986 (2 nº [?]. - [Bayonne], 30 x $21 \mathrm{~cm}$ En français. - Rédigé par des élèves du Lycée Saint-Louis Villa Pia [Bayonne]. - Voir aussi Pianotons et Petit (Le) résistant de poche

Ville de Ciboure. Bulletin municipal d'information. janv. 1973 - oct.1975 (n¹-4). Ciboure, $38 \times 26$ puis $32 \times 25 \mathrm{~cm}$

En français. - Devient: Zubiburuan

58 * Viodos-Abense. Notre commune. Bulletin municipal. Semestriel. juil. $2001\left(\mathrm{n}^{\circ} 1\right) \rightarrow$. Viodos-Abense-de-Bas, 30 × $21 \mathrm{~cm}$ En français. - Suite de: Commune de Viodos-Abense. Bulletin...

59 * Visséboulon. [janv.1978] - [...]. - [Bayonne], 30 x $21 \mathrm{~cm}$ En français. - Rédigé par des élèves du Lycée technique Paul Bert [Bayonne]

60 Vivre à Bassussarry. Irrégulier. [oct.1990] - avr.1994 (n¹-13). - Bassussarry, 30 x $21 \mathrm{~cm}$ Le $n^{\circ} 1$ ne porte pas encore le titre. - En français. - Publ. par: Vivre à Bassussarry [Bassussarry]

$61 \quad *$ Vivre à Briscous... Bimestriel. juil. $2001\left(n^{\circ} 1\right) \rightarrow$. Briscous, 30 x $21 \mathrm{~cm}$ Publ. par la Ville de Briscous. - Remplace: Beskoitze - Briscous. Bulletin municipal d'information [?] Vivre à Urrugne voir Urruñan bizi

Vivre en H.L.M.! \{Bulletin du\} Comité de défense des locataires de Bayonne. Irrégulier. [1980] ( $\left.{ }^{\circ} 1\right)-1983$ [?]. - [Bayonne], 30 x $21 \mathrm{~cm}$ En français Vivre et entreprendre à Anglet. janv. - 7 mars 1989 ( $\left.n^{\circ} 1-5\right)$ [?]. - Anglet, 30 x 21 puis $41 \times 32 \mathrm{~cm}$ En français. - Publ. à l'ocassion des élections municipales de mars 1989 pour soutenir la liste "Vivre et entreprendre à Anglet » Vivre Urruñan bizi voir Urruñan bizi

Voie d'avenir. Bulletin d'information des cellules des cheminots bayonnais [puis Journal des cellules des cheminots de Bayonne] du Parti communiste français. 1971 - [...]. [Bayonne], $31 \times 21$ puis $27 \times 21 \mathrm{~cm}$ Paraissait encore en 1974. - En français. - Suite de: Bonne (La) voie. - Devient: Flash. Bulletin des cellules du P.C.F. Cheminots de Bayonne 


\section{Impr. à Mauléon, 31 x $23 \mathrm{~cm}$}

En français. - Publ. à l'occasion des élections législatives de mars 1993 pour soutenir la candidature de Pierre Bidart

Voix de Belloc. Trimestriel. mars $1989\left(\mathrm{n}^{\circ} 132\right) \rightarrow$. Urt, 21 x $16 \mathrm{~cm}$

En français. - Publ. par: Abbaye Notre-Dame de Belloc [Urt]. - Suite de: Corde magno. - Voir aussi Abbaye N.-D. de Belloc. Lettre aux oblats

Voix (La) de François voir Patxiren botza

* Voix (La) de la cité. Cellule du Parti communiste français de Habas-la-Plaine. Bulletin d'information. [...]. - [Bayonne], 27 x $21 \mathrm{~cm}$

Paraissait en 1966. - En français

Voix (La) de St-Léon. Mensuel. [...]. - Anglet, Lille, 44 x $32 \mathrm{~cm}$

Bulletin paroissial de Saint-Léon [Anglet]. - Paraissait en 1960 et 1961. - En français. - Texte en partie identique à celui de nombreux autres bulletins paroissiaux, impr. à Lille

- janv.1993 (n¹-3). nov./déc.1993 - mars/avr.1999 (n4-32). - Bayonne puis Anglet, $30 \times 21$ ou $42 \times 30 \mathrm{~cm}$

En français. - Publ. par: Rassemblement pour la République. $5^{\mathrm{e}}$ circonscription des Pyrénées-Atlantiques [Bayonne] puis Section d'Anglet [Anglet]. - Reprend les éléments de: Rassemblement (Le). - En août 1993 certains éléments repris par: Echo (L') des cantons Irrégulier. oct.1970 ( $1^{\text {re }}$ année, $\left.n^{\circ} 1\right)$ - 1977 [?]. - Biarritz ou Biarritz, Bayonne, de 44 x 30 à $27 \times 21 \mathrm{~cm}$

Le sous-titre varie et disparaît. - En français. - Dir. publ. Georges de Styn. - Pour le suppl. voir Côte basque - magazine qui devient: Atlantique-Pyrénées

Vvrraaoouuaarr. [puis Mensuel d'informations objectives sur le sport auto... Vu par l'A.S.A. Côte basque]. [févr./mars 1977] (n¹) - [...]. - Bayonne, 30 x 21 puis 21 x $15 \mathrm{~cm}$ Paraissait encore en [août 1977] ( $\left.{ }^{\circ} 5\right)$. - En français. - Publ. par: Association sportive automobile Côte basque [Bayonne]

76 * Wazup Côte basque. Mensuel. juin $2004\left(\right.$ vol.1, $\left.\mathrm{n}^{\circ} 1\right) \rightarrow$. Anglet, $24 \times 17 \mathrm{~cm}$ En français

77 Xilo. Magazine. Bimestriel. juil. 2001 - avr./mai 2002 (n¹-5). - Bayonne, 33 x $24 \mathrm{~cm}$ Avec un $n^{\circ} 0$ de janv. 2001 et un nohors série de sept. 2001 publ. à l'occasion du 11e championnat du monde professionnel de cesta punta. - En français

78 Ximixt. [avr. 1988]. - [Saint-Jean-Pied-de-Port], $21 \times 15 \mathrm{~cm}$ Bulletin politique en forme de "fanzine». - En français; quelques textes basques. - Remplace: Apoak

79 Ximixta. [déc. 1987 - mai 1988] (2 nº [?]. - [Cambo-les-Bains], 30 x $21 \mathrm{~cm}$ En basque. - Publ. par: Xalbador ikastegia [Cambo-les-Bains]. - Voir aussi Pindarra; Hats; Nahiz et Hator

80 Xirika. Gazteon kazeta. Hebdomadaire. 5/11 févr. $2004\left(n^{\circ} 1\right) \rightarrow$. Senpere, $42 \times 19 \mathrm{~cm}$ Avec un $n^{\circ} 0$ de janv. 2004. - En basque

81 Xirrixta. Mensuel. oct. 1992 - avr. 1998 ( $\left.\mathrm{n}^{\circ} 1-61\right)$ - - Uztaritze puis Hendaia puis Hendaia, Irun, $24 \times 22 \mathrm{~cm}$ 
Illustré pour les enfants. - Avec un spéc. d'avr. 1992. - En basque. - Publ. par: Euskal kultur erakundea; en français: Institut culturel basque [Ustaritz]; puis Xirrixtazaleak elkartea [Hendaye] puis Xirrixtazaleak elkartea et Letrazopa elkartea [Irun]. - Constitué en partie par des dessins et des textes extraits de " Toboggan » [Toulouse, Milan-presse], traduits en basque

Xutik! Revue de contre-information sur la guerre du Golfe. Collectif Patxa. [janv 1991] ( ${ }^{\circ}$

1) [?]. - Baiona, $30 \times 21 \mathrm{~cm}$

En français. - Publ. par: Patxa [Bayonne]

Yacht-club Adour-Atlantique. \{Annuaire officiel\}.Annuel. 1981-1993. 1995-2000. Anglet, Toulouse puis Anglet, 21 x 15 puis $30 \times 21 \mathrm{~cm}$

En français. - Suite de: Plaisance Adour-Atlantique et Sud-Gascogne

Yacht-club (Le) Adour-Atlantique. Revue trimestrielle. juil. 1969-oct. 1970 (n¹-6). Anglet, $27 \times 21 \mathrm{~cm}$

En français. - Devient: Plaisance Adour-Atlantique et Sud-Gascogne

Z.U.P.-liaison. Mensuel ou irrégulier. déc.1970 - déc. 1981 (n¹-67). - Bayonne, 38 x 28 ou $43 \times 30 / 32$ puis $32 \times 23 \mathrm{~cm}$

En français. - De 1973 à 1980 publ. successivement par: Groupe d'animation de la Z.U.P. des Hauts de Sainte-Croix [Bayonne] puis Mission d'éducation permanente [Bayonne] puis office régional d'éducation permanente [Bayonne]

Zabal. Bimestriel. mai/juin 1973 - juil./août 1976 ( $\left.{ }^{\circ}{ }^{\circ} 1-19\right)$. - Baiona, 30 x 21 puis 24 x $16 \mathrm{~cm}$ En basque

Zachée. Le journal de la Pastorale des jeunes du Pays basque. Trimestriel. mars/mai [1999] - juin/août [2000] (n¹-6). - Bayonne, Blagnac, 40 x $28 \mathrm{~cm}$ Titre basque: Zakeo. Gazteak elizan. - En français; quelques textes basques

Zazpiak-bat. Bulletin de liaison des sept communes du canton d'Espelette. mars 1976 (1 n o) [?]. - Impr. à Cambo, 28 x $21 \mathrm{~cm}$

En français; un texte basque. - Publ. à l'occasion des élections cantonales de mars 1976 pour soutenir la candidature de Michel Labéguerie

* Zazpiak bat. Bulletin extérieur d'information, publié par E.MA. (Mouvement abertzale de gauche). déc.1988 [?] - [...]. - Bayonne

Il aurait paru $3 n^{\circ}$ [?]. - Publ. par: Ezkerreko mugimendu abertzalea (E.MA.) [Bayonne]. - Voir aussi Emaitza

Zeiñützülian voir Autour du clocher

Zer berri. Revue d'information[s] du Centre hospitalier \{de la\} Côte basque. $3 n^{\circ}$ par an. avr. $1995\left(\mathrm{n}^{\circ} 1\right) \rightarrow$. Bayonne, 30 x 21 puis 42 x $30 \mathrm{~cm}$

Avec un no de [déc.1994]. - Titre français: Quoi de neuf ?. - En français. - Voir aussi Entre nous [2002]; Infos première et Qualiscope

Zerbitzari. Irrégulier. 1980 - [mars 2004]. - [Hasparren], 35 x 26 puis 30 x $21 \mathrm{~cm}$

Titre français: Au service de tous. - En français; quelques textes basques. - Publ. à l'occasion des élections cantonales de 1980 à 2004 pour soutenir la candidature de Jacques Coumet, et à l'occasion des élections municipales de 1995 et 2001 pour soutenir la liste "Hasparren demain - Biharko Hazparne »

Zertaco ez? voir Pourquoi pas?

Zerua Euskal herritik ikusirik voir Ciel (Le) au Pays basque

Ziberoko bizia voir Miroir (Le) de la Soule... 
* Ziburu-Ciboure. Bulletin [puis Magazine] d'information de la mairie. Irrégulier. janv. $2002 \rightarrow$. Ciboure, $30 \times 21 \mathrm{~cm}$

En français. - Suite de: Ciboure. Bulletin municipal

Zirika. Donibane-Lohizune herriko talde abertzalearen informazio aldizkaria [puis Donibane-Lohizune-ko aldizkaria]. Bimestriel. $1^{\mathrm{er}}$ avr. $2000\left(\mathrm{n}^{\circ} 1\right) \rightarrow$. Donibane-Lohizune, $30 \times 21 \mathrm{~cm}$

En français. - Publ. par: Zirika [Saint-Jean-de-Luz]. - Reprend certains éléments de: Kitzik

98

Zirta. Gazte abertzaleen batasun euskaldunaren aldizkaria. [nov. 1989 - nov. 1990] (n¹-5 ) [?]. - Ziburu, 30 × $21 \mathrm{~cm}$

En français puis en basque et français. - Publ. par: Gazte abertzaleen batasun euskalduna (G.A.B.E.) [Ciboure]. - Voir aussi Atzar

Zitona. [puis Journal de l'Ecole de Mendionde-Gréciette]. Annuel. [1977/78] [?] - juin 1985 ( $\left.{ }^{\circ} 1-8\right)$. juin 1986. juin 1988. - Mendionde, 21 x 15 puis 21 x 30 puis $15 \times 21 \mathrm{~cm}$ En français

Zubiac. Bimestriel. nov./déc. $2004\left(\mathrm{n}^{\circ} 1\right) \rightarrow$. Bayonne, 30 x $21 \mathrm{~cm}$

En basque et français. - Publ. par: Eusko alkartasuna Iparralde [Bayonne]. - Suite de: Alkarrekin lan

Zubiburuan. Périodique [puis Bulletin] d'information communale. Irrégulier. avr./ mai 1977 - janv. 1979 ( $\left.{ }^{\circ} 1-6\right)$. - Ciboure, 30 x $21 \mathrm{~cm}$

En français. - Suite de: Ville de Ciboure. Bulletin municipal d'information. - En 1984 devient: Bulletin municipal. Ciboure

\section{Zuraideko berriak voir Souraïde. Bulletin municipal}

Zutik! L.K.I.-ren aldizkaria [puis Bulletin de la Section du Pays basque de la L.C.R. [Ligue communiste révolutionnaire] - Quatrième Internationale]. Supplément à Rouge. 1988 1989 [?]. - Bayonne, 30 x $21 \mathrm{~cm}$

En français. - Reprend les éléments de: Satorra. - Devient: Lettre rouge

\section{INDEX}

Index chronologique : 20e siècle

Index géographique : Pays basque (France)

Thèmes : bibliographie

Mots-clés : publication périodique 